\title{
Possible Role of the Pineal Gland in Pituitary Prolactin Secretion in Female Rats
}

\author{
Keirchi Minato, Katsuyuki Takahashi, * Nobuko \\ Ikeno, ${ }^{*}$ Masaaki Watanabe, ${ }^{*}$ Hiroshi Endo* and \\ Hironao Yamamoto* \\ Department of Obstetrics and Gynecology, Tohoku University \\ School of Medicine, Sendai 980 and *Department of \\ Obstetrics and Gynecology, Sendai National Hospital, \\ Sendai 980
}

\begin{abstract}
Minato, K., Takahashi, K., Ineno, N., Watanabe, M., Endo, H. and Yamamoto, H. Possible Role of the Pineal Gland in Pituitary Prolactin Secretion in Female Rats. Tohoku J. exp. Med., 1984, 143(3), 305-313—Wistar female rats housed under conditions of $12 \mathrm{hr}$ dark/12 hr light were pinealectomized (PX) or underwent sham-operation (SO) 21 days after ovariectomy, on the 7 th or on the 15-17 th day of pregnancy. Serum and pituitary prolactin (PRL) levels in ovariectomized (OVX) rats were determined 9 days after pinealectomy. In the case of OVX rats receiving estrogen and progesterone injections (OVX-EP), PRL levels were determined $48 \mathrm{hr}$ after injection administered 7 day after pinealectomy. PRL levels in pregnant rats were determined on the 20th day of pregnancy and in postpartum rats, on the 3rd day following parturition. As compared with the SO control, pinealectomy resulted in a significant decrease in the serum PRL level in the OVX-EP rats but in a significant increase in that level in the OVX, pregnant and postpartum rats. In OVX-EP rats, exogenous estrogen raised the serum PRL level less in PX than in SO rats, probably because the pineal gland is closely related to the facilitation of PRL secretion by estrogen. The high estrogen level in OVX-EP rats seemed to trigger pineal stimulation of PRL release, but low estrogen levels in OVX and postpartum rats or markedly high levels of progesterone in pregnant rats on the 20th day are thought to cause pineal inhibition. - prolactin; pineal gland; estrogen; progesterone
\end{abstract}

There have been no uniform conclusions about the role of the pineal gland in pituitary prolactin (PRL) secretion in the rat, because different experimental conditions have resulted in either inhibition or facilitation of PRL release by the pineal gland. We have previously studied the change in PRL secretion after pineal removal and administration of pineal bioactive substances, such as melatonin, serotonin and arginine vasotocin (AVT) and have demonstrated that antagonistic mechanisms of the pineal gland which affect pituitary PRL secretion

Received for publication, November 7, 1983. 
exist in the pineal-hypothalamo-pituitary interaction. We have suggested that the pineal gland can either facilitate or inhibit pituitary PRL secretion depending on changes in predominance of these mechanisms (Minato and Takahashi 1982 ; Minato et al. 1983).

Participation of the pineal gland in the regulation of the gonadal function has been firmly established. Because levels of estrogen and progesterone in blood rise after pinealectomy in rats (Vaughan et al. 1978; Nir and Hirschmann 1980), these ovarian hormone levels are thought to be controlled to some degree by the pineal gland. On the other hand, there are specific estradiol binding sites in the pineal gland as well as in the pituitary gland (Cardinali 1977). It is generally known that estrogen causes a rise in blood level of PRL. This action of estrogen is explained by evidence that this hormone increases pituitary PRL biosynthesis (Lieberman et al. 1978; Maurer 1982), and that it depresses the release of dopamine in the hypothalamus. However, the mechanism of the pineal gland concerned with the increase of PRL due to the effect of estrogen has not been clarified. In the present study, we have investigated what changes are caused by pinealectomy in the levels of serum and pituitary PRL in various reproductive states of rats, namely, ovariectomized rats, ovariectomized estrogen and progesterone primed rats, pregnant rats and postpartum rats. From our results and the observations of others, we conclude that a) changes in the reproductive state have an influence on pineal functions, which in turn has an effect on pituitary PRL secretion, b) the pineal gland is closely related to PRL release stimulated by estrogen as well as the hypothalamus and the pituitary, and c) sex steroid hormone may be concerned with this alteration of pineal functions.

\section{Materials and Methods}

\section{Animals}

Female Wistar rats ( 10 weeks old, $200 \pm 20 \mathrm{~g}$ ) were used in all experiments. They were housed with a $12 \mathrm{hr}$ light/12 hr dark photoperiod, maintained at a temperature of $22 \pm 2^{\circ} \mathrm{C}$, and provided with food and water ad libitum.

Ovariectomized rats. Rats were ovariectomized (OVX) in order to eliminate any influence of sex cycle on PRL secretion. Subsequently, they were pinealectomized (PX) according to the modified method of Kuszak and Robin (1977) or were subjected to a shamoperation (SO) 21 day after ovariectomy. Nine days after pinealectomy or sham-operation, they were decapitated in order to determine serum and pituitary PRL levels.

Ovariectomized estrogen and progesterone primed rats. Rats were pinealectomized or subjected to sham-operation 21 days after ovariectomy. Seven days after the operation, they were injected intramuscularly with $50 \mu \mathrm{g}$ estrogen and $2 \mathrm{mg}$ progesterone (OVX-EP) and were decapitated $48 \mathrm{hr}$ after the steroid treatment.

Late pregnant rats. After rats were mated, the morning on which a sperm-positive vaginal smear was noted was termed the 1st day of pregnancy. They were subjected to pinealectomy or sham-operation on the 7th day of pregnancy. They were decapitated on the 20th day of pregnancy.

Early postpartum rats. Rats were subjected to pinealectomy or sham-operation on the 15th to 17th day of pregnancy. They were delivered of their litters in separate cages, allowed 
to suckle until the 3 rd day of postpartum, and then decapitated 30 min after separation from their litters in order to avoid the influence of suckling.

\section{Preparation for radioimmunoassay}

Blood samples and pituitaries were collected between 15:00-17:00. Blood samples were centrifuged at 3,000 rpm for $15 \mathrm{~min}$ and sera were obtained. A pituitary gland was homogenized in $4.0 \mathrm{ml}$ physiological saline and centrifuged at 2,000 rpm for 5 min to obtain supernatant. The sera and pituitary homogenates were stored at $-20^{\circ} \mathrm{C}$. PRL contents were determined by radioimmunoassay and expressed in terms NIAMDD rat PRL-RP-1 $(11.0 \mathrm{iu} / \mathrm{mg})$. Significance was calculated according to the Student's $t$-test. PRL levels were given by mean \pm s.e.

\section{RESUlts}

\section{OVX rats}

In OVX rats without EP treatment, pinealectomy raised serum PRL levels and lowered pituitary PRL concentrations as follows: Serum PRL levels of PX rats $(128.9 \pm 18.9 \mathrm{ng} / \mathrm{ml})$ were significantly $(p<0.001)$ higher than those of SO rats $(47.9 \pm 5.0 \mathrm{ng} / \mathrm{ml})$ (Fig. la). Pituitary PRL levels of PX rats $(831.0 \pm 109.6 \mathrm{ng} /$ mg AP) (AP : anterior pituitary) were significantly $(p<0.001)$ lower than those of $\mathrm{SO}$ rats $(1438.3 \pm 120.7 \mathrm{ng} / \mathrm{mg} \mathrm{AP})$ (Fig. 1b).

\section{OVX-EP rats}

In OVX-EP rats, pinealectomy markedly $(p<0.001)$ suppressed serum PRL
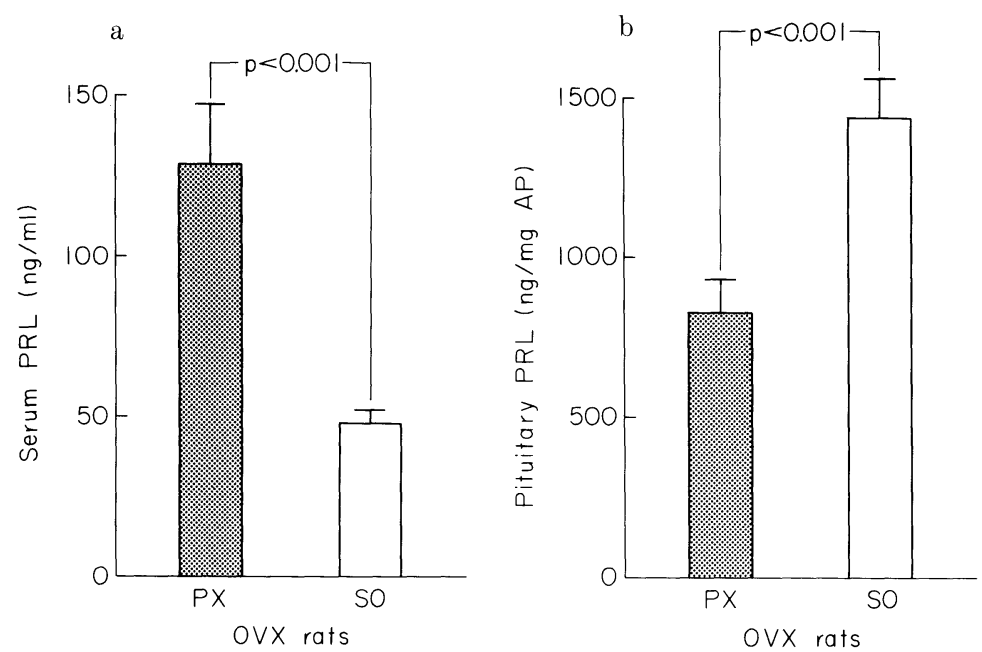

Fig. 1. Serum and pituitary PRL levels of ovariectomized (OVX) rats after pinealectomy (PX) or sham-operation (SO). Rats were pinealectomized or underwent sham-operation 21 days after ovariectomy. Serum and pituitary PRL levels were determined 9 days after pinealectomy or sham-operation. The number of samples was 6 .

a: Serum PRL levels were compared between PX and SO rats.

b : Pituitary PRL levels were compared between PX and SO rats. 

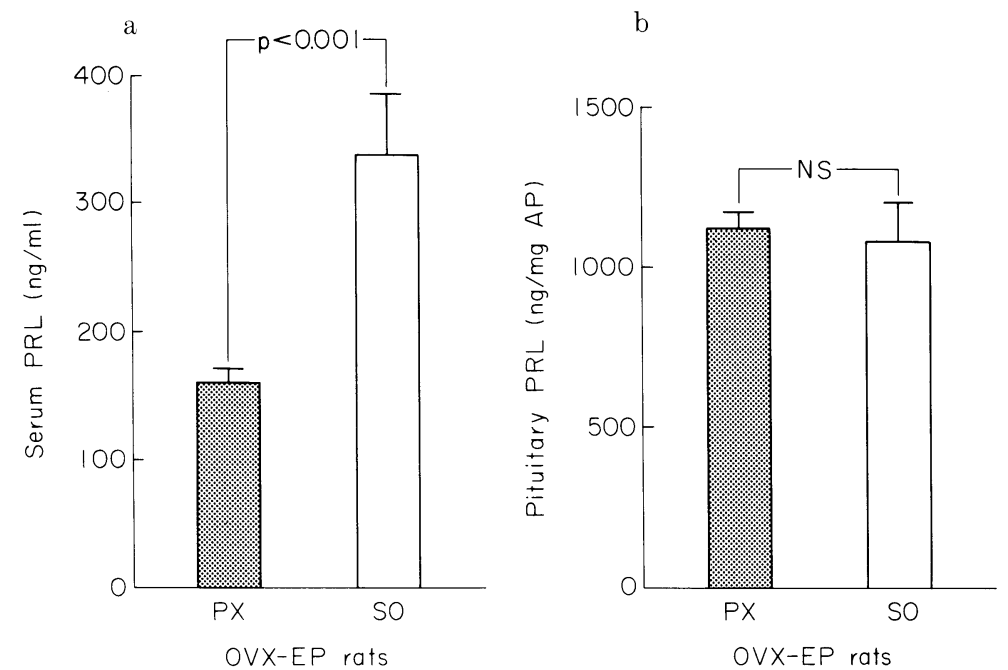

Fig. 2. Serum and pituitary PRL levels of ovariectomized estrogen and progesterone primed (OVX-EP) rats after pinealectomy (PX) or sham-operation (SO). Rats were pinealectomized or underwent sham-operation 21 days after ovariectomy, and injected with $50 \mu \mathrm{g}$ estrogen and $2 \mathrm{mg}$ progesterone 7 days after pinealectomy or sham-operation. Serum and pituitary PRL levels were determined $48 \mathrm{hr}$ after the injections. The number of samples was 6 .

a: Serum PRL levels were compared between PX rats and SO rats.

b : Pituitary PRL levels were compared between PX rats and SO rats.
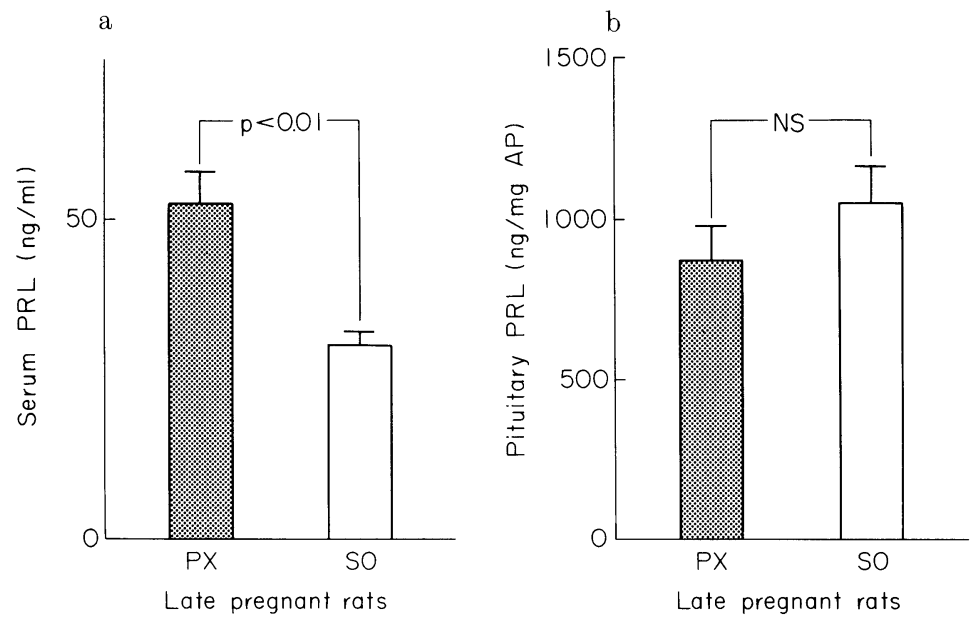

Fig. 3. Serum and pituitary PRL levels of late pregnant rats after pinealectomy (PX) or sham-operation. Rats were pinealectomized or underwent sham-operation on the 7th day of pregnancy. Serum and pituitary PRL levels were determined on the 20th day of pregnancy. The number of samples was 6 .

a : Serum PRL levels were compared between PX rats and SO rats.

b : Pituitary PRL levels were compared between PX rats and SO rats. 

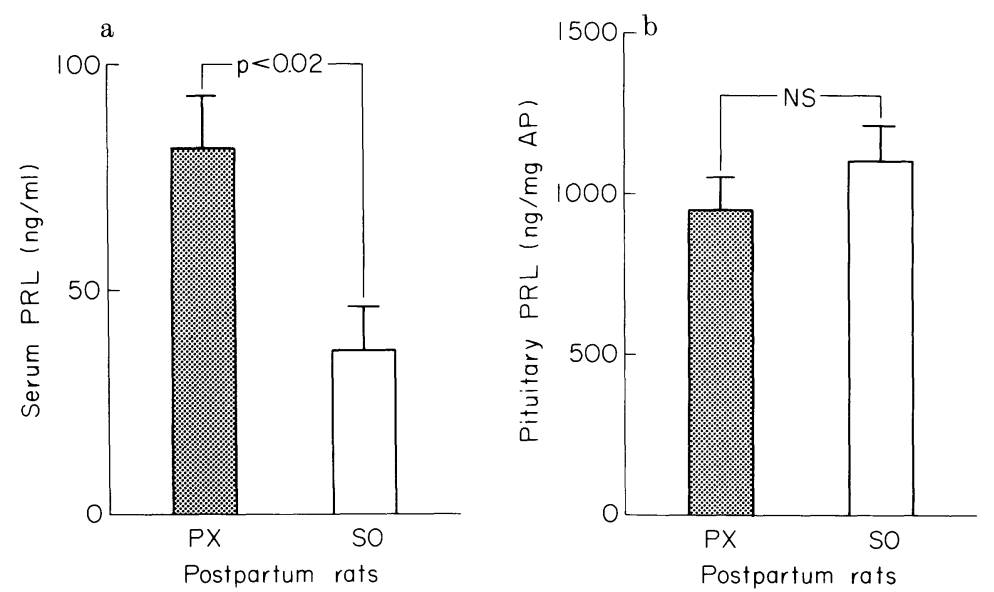

Fig. 4. Serum and pituitary PRL levels of postpartum rats after pinealectomy (PX) or sham-operation (SO). Rats were pinealectomized or underwent sham-operation on the 15th to 17th day of pregnancy. Serum and pituitary PRL levels were determined on the 3rd day after parturition.

The number of samples was 6 .

a: Serum PRL levels were compared between PX rats and SO rats.

b : Pituitary PRL levels were compared between PX and SO rats.

levels $(158.8 \pm 12.1 \mathrm{ng} / \mathrm{ml})$ as compared to sham-operation $(338.6 \pm 47.1 \mathrm{ng} / \mathrm{ml})$ (Fig. 2a). However, there was no significant difference in pituitary PRL levels between PX rats $(1118.0 \pm 52.8 \mathrm{ng} / \mathrm{mg} \mathrm{AP})$ and $\mathrm{SO}$ rats $(1077.0 \pm 126.8 \mathrm{ng} / \mathrm{mg} \mathrm{AP})$ (Fig. 2b).

\section{Late pregnant rats}

Pinealectomy resulted in a significant $(p<0.01)$ increase in serum PRL levels of PX rats $(52.4 \pm 5.0 \mathrm{ng} / \mathrm{ml})$ as compared to the levels of SO rats $(30.5 \pm 1.7 \mathrm{ng} /$ ml) (Fig. 3a). However, pituitary PRL levels of PX rats $(888.4 \pm 89.3 \mathrm{ng} / \mathrm{mg} \mathrm{AP})$ did not significantly differ from the levels of SO rats $(1024.8 \pm 137.9 \mathrm{ng} / \mathrm{mg}$ AP) (Fig. 3b).

\section{Early postpartum rats}

Pinealectomy significantly enhanced serum PRL levels of postpartum rats on the 3rd day after parturition. Serum levels were significantly $(p<0.02)$ higher in PX rats $(81.6 \pm 11.6 \mathrm{ng} / \mathrm{ml})$ than in $\mathrm{SO}$ rats $(36.7 \pm 9.8 \mathrm{ng} / \mathrm{ml})$ (Fig. $4 \mathrm{a})$. Pituitary PRL levels, however, did not significantly differ between PX rats (952.1 \pm $99.6 \mathrm{ng} / \mathrm{mg} \mathrm{AP}$ ) and SO rats (1095.5 $\pm 61.7 \mathrm{ng} / \mathrm{mg} \mathrm{AP)} \mathrm{(Fig.} 4 \mathrm{~b})$.

Discussion

The pineal gland has been demonstrated to influence PRL secretion, the effect being either stimulatory or inhibitory in the rats. The present study has also demonstrated the existence of opposite functions of the pineal gland in relation to 
levels of pituitary PRL in various reproductive states. Pinealectomy brought about a decrease in serum PRL levels of OVX-EP rats but an increase in such levels in OVX, late pregnant (the 20th day) and postpartum rats. This suggests that alternate mechanisms of the pineal gland, mentioned previously, regulate rat PRL secretion. Because the levels of the ovarian hormones significantly vary during these reproductive states, the hormones seem to be related to the alternation of the PRL regulating mechanisms of the pineal gland.

Biosynthesis of pineal peptides in the rat pineal gland is affected by the ovarian steroid hormone; estrogen raises but progesterone lowers this biosynthesis (Cardinali and Vacas 1978). AVT, one of the pineal peptide hormones, markedly stimulates PRL release and is also increased by estrogen (Cardinali et al. 1974). It is well known that estrogen facilitates PRL synthesis in the pituitary. Additionally, this ovarian hormone may indirectly elevate circulating PRL levels via AVT. Our results support this hypothesis, because estrogen enhanced serum PRL levels less in OVX-PX rats than those in OVX-SO rats. The pineal gland is apparently related to the PRL increasing effect of estrogen. In other words, it can be suggested that the intact pineal gland is necessary for exogenous estrogen to raise the level of PRL.

Estrogen is reported not to affect biosynthesis of melatonin in the pineal gland (Pang and Ralph 1975; Wurtman and Ozaki 1978). We have previously suggested that melatonin has not only a stimulatory effect but also an inhibitory effect on rat PRL secretion, although the inhibition is usually masked by AVT (Minato et al. 1983), inasmuch as this peptide is a potent stimulant of PRL (Pavel 1978; Minato and Takahashi 1982; Minato et al. 1983) and is released by melatonin (Pavel et al. 1975). Therefore, we have hypothesized that the pineal inhibitory effect of melatonin would be predominant over the stimulatory effect, when the AVT level is reduced by low estrogen level or a considerably high level of progesterone.

Levels of estrogen and progesterone are low in OVX and postpartum rats and high in OVX-EP rats, but vary daily in the late pregnancy in rats (Nir et al. 1979). According to our hypothesis described above, neither a low level of estrogen nor a significantly high level of progesterone increases AVT, and this results in an amplification of the pineal inhibitory mechanism in rats. Pinealectomy may eliminate this inhibition and enhance PRL secretion. Indeed, we have observed that serum PRL level rose after pinealectomy in OVX and postpartum rats in which serum estrogen and progesterone decreased. Pinealectomy has also been reported to result in an increase in serum PRL levels in blind-anosmic rats (Leadem and Blask 1982a,b). It is well known that atrophy of the ovary is brought about 8 weeks after surgically induced blind-anosmia. The pineal inhibition of PRL in these rats which have low levels of the ovarian hormones seems to support our result observed in OVX rats.

On the other hand, the effect of a high level of estrogen on pineal function 
would be opposite, since the stimulatory function of the pineal gland is the major factor in OVX-EP rats. In the present study, OVX rats were treated with $50 \mu \mathrm{g}$ estrogen and $2 \mathrm{mg}$ progesterone. However, $50 \mu \mathrm{g}$ estrogen is sufficient to increase AVT, nagating the inhibitory effect of progesterone (Cardinali and Vacas 1978). Therefore, the serum PRL level was depressed by pinealectomy in OVX-EP rats, in which the pineal gland has a stimulatory effect. In androgenized female rats subjected to ovariectomy and injection of estrogen, pinealectomy also resulted in a decrease in plasma PRL level (Gala et al. 1983). Exogenous estrogen in these rats was demonstrated to induce the diurnal PRL pattern and seems to cause the pineal facilitation of PRL release as observed in OVX-EP rats.

It has been reported that serum levels of estrogen increase with the length of pregnancy but that progesterone concentration drastically decreases on the 22nd day of pregnancy in intact rats (Nir et al. 1979), PX rats and SO rats (Nir and Hirschmann 1980). According to Nir and Hirschmann, progesterone levels are about 2000-times and 1000-times higher than estrogen levels on the 20th and 21st day of pregnancy in PX rats and SO rats. These considerably high levels of progesterone on the 20 th and 21st day can partially explain PRL inhibition by the pineal gland on the 20 th day of pregnancy, because on those days AVT synthesis is suspected to be suppressed by progesterone. Furthermore, it was observed in this study that pinealectomy actually resulted in an increase in serum PRL level on the 20th day of pregnancy. By contrast, under an increased level of estrogen and a decreased level of progesterone on the 22nd day of pregnancy the serum PRL level must be decreased by pinealectomy, since pineal removal eliminates the PRL releasing mechanism activated by increased estrogen. Indeed, Nir and Hirschmann (1982) have indicated that pinealectomy significantly decreased serum PRL levels on the 22nd day of rat pregnancy but had an insignificant effect on the 21st day. These changes of the estrogen and progesterone concentrations during this period seem to adequately explain the different influences of pinealectomy on PRL secretion.

Results of the present investigation as well as our previous study suggest that the rat pineal gland regulates PRL secretion by alternate mechanisms. This may be related to the balance of melatonin and AVT depending on the ratio of the ovarian hormone levels, namely on the competitive effects of estrogen and progesterone on the pineal peptide biosynthesis. Recently, Zisapel et al. (1983) have demonstrated that inhibition of dopamine release by melatonin in the rat hypothalamus significantly changes during the estrous cycle in vitro and that a low level of melatonin only slightly inhibits the release of dopamine in diestrous. Because dopamine can markedly depress PRL secretion, their results suggest that the change in the effect of melatonin on PRL release depends on alteration of the ovarian hormone level. This may partially support our hypothesis. However, AVT immunoactivity in the rat pineal gland has been reported to increase dramatically in August (Prechel et al. 1983). Although the present study was 
performed taking the seasonal and diurnal rhythms of the pineal hormones into consideration, the further investigation would be required to define the role of the pineal gland in pituitary PRL secretion.

\section{Acknowledgment}

We are grateful to Prof. Masakuni Suzuki for his kind advice. We would also like to thank NIAMDD, NIH for the materials used in the rat PRL radioimmunoassay.

\section{References}

1) Cardinali, D.P. (1977) Nuclear receptor estrogen complex in the pineal gland: modulation by sympathetic nerves. Neuroendocrinology, 24, 333-346.

2) Cardinali, D.P. \& Vacas, M.I. (1978) Feedback control of pineal function by reproductive hormones - a neuroendocrine paradigm. J. neural Transmission, Suppl. 13, $175-201$.

3) Cardinali, D.P., Nagle, C.A. \& Rosner, J.M. (1974) Effect of estradiol on melatonin and protein synthesis in the rat pineal organ. Hormone Res., 5, 304-310.

4) Gala, R.R., Haisenleder, D.J. \& Pieper, D.R. (1983) Influence of blinding, olfactory bulbectomy and pinealectomy on plasma prolactin levels in the neonatally androgenized female rat. Neuroendocrinology, 37, 9-13.

5) Kuszak, Y. \& Robin, M. (1977) A new technique of pinealectomy for adult rats. Experientia, 33, 283-284.

6) Leadem, C.A. \& Blask, D.E.(1982a) A comparative study of the effects of the pineal gland on prolactin synthesis, storage and release in male and female blind-anosmic rats. Biol. Reprod., 26, 413-421.

7) Leadem, C.A. \& Blask, D.E. (1982b) Pineal gland inhibition of prolactin cell activity is independent of gonadal regression. Neuroendocrinology, 35, 133-138.

8) Lieberman, M.E., Maurer, R.A. \& Gorski, J. (1978) Estrogen control of prolactin synthesis in vitro. Proc. nat. Acad. Sci. USA, 75, 5946-5949.

9) Maurer, R.A. (1982) Estradiol regulates the transcription of the prolactin gene. $J$. biol. Chem., 257, 2133-2136.

10) Minato, K. \& Takahashi, K. (1982) The role of the pineal gland on the regulating system of hypophysial prolactin secretion - the prolactin-releasing and release inhibiting action of the rat pineal gland. Fol. endocr. jap., 58, 945-961. (Japanease)

11) Minato, K., Takahashi, K., Ikeno, N., Watanabe, M., Endo, H. \& Yamamoto, H. (1983) Evidence for prolactin-releasing and release-inhibiting effects of melatonin, serotonin and arginine vasotocin. Tohoku J. exp. Med., 141, 107-116.

12) Nir, I. \& Hirschmann, N. (1980) The effect of pinealectomy on serum progesterone and oestradiol levels during the last stage of pregnancy in rats. Experientia, 36, 259-260.

13) Nir, I. \& Hirschmann, N. (1982) Darkness-elicited changes in rat reproduction not mediated by pineal gland. Hormone Res., 16, 385-391.

14) Nir, I., Hirschmann, N., Goldhaber, G. \& Shani, J. (1979) Pinealectomy-induced changes in blood and pituitary luteinizing hormone and prolactin levels during the last phase of pregnancy in rats. Neuroendocrinology, 28, 44-51.

15) Pang, S.F. \& Ralph, C.L. (1975) Pineal and serum melatonin at midday and midnight following pinealectomy or castration in male rats. $J$. exp. Zool., 193, 275-280.

16) Pavel, S. (1978) Arginine vasotocin as a pineal hormone. J. neural Transmission, Suppl. 13, 135-155.

17) Pavel, S., Calb, M. \& Georgescu, M. (1975) Reversal of the effects of pinealectomy on the pituitary prolactin content in mice by very low concentrations of vasotocin injected into the third cerebral ventricle. J. Endocr., 66, 289-290. 
18) Prechel, M.M., Audhya, T.K. \& Schlesinger, D.H. (1983) A seasonal variation in arginine vasotocin immunoactivity in rat pineal gland. Endocrinology, 112, 14741478.

19) Vaughan, G.M., Reiter, R.J., Siler-Khodr, T.M., Sackman, J.W., Allen, J.P., Vaughan, M.K., McGuire, W.L., Johnson, L.Y. \& Starr, P. (1978) Influence of pinealectomy on serum estrogen and progesterone levels in blind-anosmic female rats. Experientia, 34, 1378-1379.

20) Wurtman, R.J. \& Ozaki, Y. (1978) Physiological control of melatonin synthesis and secretion: Mechanisms generating rhythms in melatonin, methoxytryptophol, and arginine vasotocin levels and effects on the pineal of endogenous catecholamines, the estrous cycle, and environmental lighting. $\quad J$. neural Transmission, Suppl. 13, 59-70.

21) Zisapel, N., Egozi, Y. \& Laudon, M. (1983) Inhibition by melatonin of dopamine release from rat hypothalamus in vitro: variations with sex and the estrous cycle. Neuroendocrinology, 37, 41-47. 